\title{
Simulation als Bestandteil eines BIM-basierten Vorgehens zur Planung der Baustellenlogistik im Großanlagenbau
}

\author{
Jana Stolipin ${ }^{1 *}$, Ulrich Jessen ${ }^{1}$, Jan Weber ${ }^{2}$, Sigrid Wenzel ${ }^{1}$, Markus König ${ }^{2}$ \\ ${ }^{1}$ Institut für Produktionstechnik und Logistik, Fachgebiet Produktionsorganisation und Fabrikplanung, Universität Kassel, \\ Kurt-Wolters-Straße 3, 34125 Kassel; *jana.stolipin@uni-kassel.de. \\ ${ }^{2}$ Fakultät für Bau- und Umweltingenieurwissenschaften, Lehrstuhl für Informatik im Bauwesen, Ruhr-Universität Bochum, \\ Universitätsstraße 150, Gebäude IC, 44780 Bochum.
}

\begin{abstract}
Kurzfassung. Für die Konstruktions- und Montageplanung von Großanlagen hat sich eine digitale modellbasierte Arbeitsweise, auch als Building Information Modeling (BIM) bezeichnet, durchgesetzt. Dieser Beitrag stellt für diesen Anwendungskontext ein Vorgehensmodell zur integrierten Planung und Gestaltung von logistischen Prozessen auf einer Baustelle vor. Ausgehend von einem BIM-basierten digitalen Modell und mit Unterstützung einer fachspezifischen Ontologie und Beschreibungen von Standardprozessen wird die Planung der Baustellenlogistik durchgeführt und mittels Simulation überprüft. Anhand eines Beispielmodells werden die einzelnen Schritte des Vorgehensmodells vorgestellt, das Planungsergebnis simulativ überprüft und die Ergebnisse abschließend diskutiert.
\end{abstract}

\section{Einleitung}

E ffiziente und wirtschaftliche Produktions- und L ogistikprozesse sind für den Unternehmenserfolg essentiell [1]. Ihrer Planung und Gestaltung wird damit eine hohe Relevanz zugesprochen; die geforderte hohe Planungsqualität bei gleichzeitig hoher Planungssicherheit auch bei komplexen Systemen kann durch die A nwendung digitaler Planungsmethoden und -modelle erreicht werden [2]. A uch für die logistischen Prozesse auf einer B austelle im Großanlagenbau ist eine zuverlässige Planung entscheidend, da nur auf diese W eise eine abgesicherte Terminierung aller Bauprozesse auf dem Baustellengelände und ein konfliktfreier A blauf umgesetzt werden können.

Heute werden im A nlagenbau digitale M odelle insbesondere zur Entwurfsplanung eingesetzt, jedoch können diese nicht direkt zur Planung und Steuerung der B austellenlogistik genutzt werden, da beispielsweise die logistikrelevanten Informationen einzel ner A nlagekomponen- ten fehlen oder die vorhandenen Informationen nicht einheitlich strukturiert und somit nicht automatisiert auswertbar sind. Erst wenn zu jedem Bauelement die logistikrelevanten Informationen (z. B. Transport-, Lagerund M ontagehinweise) vorliegen, kann eine Planung der L ogistikprozesse auf der B austelle real isiert werden. Das digitale L ogistikmodell kann dann analysiert und im Hinblick auf ausgewählte Zielgrößen (z. B. Lagerbelegung oder Ressourcenauslastung) überprüft und ggf. verbessert werden. Ein derartiges digitales Planungsmodell kann u. a. auch als Grundlage für die Steuerung der $B$ austellenlogistik eingesetzt werden und die Entscheidungen zur operativen Durchführung der Logistikprozesse auf der B austelle unterstützen. D azu müssen allerdings die aktuellen Baufortschrittsinformationen regelmäßig gepflegt werden [3].

Der B eitrag stellt ein BIM -basiertes V orgehen zur digitalen Planung der Baustellenlogistik im Großanlagenbau vor. Das Ziel dieses V orgehens ist, ausführenden U nternehmen Hilfsmittel zur effizienten Organisation und zur Sicherstellung der Bauprozesse der A nlage vor Ort anzubieten; dabei wird zur A bsicherung der BIM -basierten Planung die Simulation eingesetzt. In diesem $B$ eitrag wird - ausgehend vom Stand der Forschung zur B austellenplanung im ersten $A$ bschnitt - im zweiten A bschnitt das BIM - basierte $\mathrm{V}$ orgehen vorgestellt. $\mathrm{N}$ eben der $\mathrm{D}$ arstellung der einzelnen Elemente des $V$ orgehens wird auch die Rolle der Simulation zur V alidierung der für eine Baustelle geplanten Logistikprozesse im Großanlagenbau erläutert. Der dritte A bschnitt konzentriert sich auf die B eschreibung der U msetzung des $V$ orgehens anhand eines $A$ nw endungsbeispiels mit besonderem Fokus auf die $V$ alidierung der dazugehörigen Planung mittels eines mit dem Simulationswerkzeug A nyL ogic erstel Iten 
Simulationsmodells. Nach der Darstellung der Simulationsergebnisse werden im abschließenden vierten $A b$ schnitt weitere Einsatzmöglichkeiten der Simulation im Rahmen der M ethodik diskutiert und potenzielle Forschungsfragen abgel eitet.

\section{Baustellenlogistikplanung}

Die Bauwirtschaft ist durch einen sehr hohen Kostendruck getrieben und gilt als eine eher konservative $B$ ranche [4]. Im Großanlagenbau (z. B. K raftwerke und Chemieanlagen) ähneln die Rahmenbedingungen in vielen A spekten denen des klassischen B aubetriebs (z. B. Hochund Tiefbau), jedoch mit dem Unterschied, dass die M ontage in B ezug auf die L ogistik eine zentrale Stellung einnimmt. L ogistik und M ontage im Großanlagenbau stellen ein komplexes und ineinander verzahntes System dar [5]. Die Baulogistik lässt sich in die drei B ereiche Versorgungs- oder Beschaffungslogistik, B austellen- oder Produktionslogistik und Entsorgungslogistik unterteilen, wobei die Baustellenlogistik alle logistischen A ufgaben auf dem B austellengelände umfasst [2]. Die B austellenlogistik im Großanlagenbau hängt u. a. von der geplanten A nlage, der Lage des Bauplatzes und den verfügbaren Ressourcen ab und stellt einen Schlüsselfaktor für den Projekterfolg dar [6].

\subsection{Praxis der Logistikplanung}

Planung und Steuerung der Prozesse auf der B austelle basieren vorwiegend auf dem $W$ issen der erfahrenen $M$ itarbeitenden [7]. Dies führt häufig zu nicht standardisiertem Planungsvorgehen und erschwert die Koordination der K ommunikation aller Projektbeteiligten [8]. B ei der Projektplanung im B auw esen ist meist nur wenig Zeit für die A rbeitsvorbereitung vorgesehen, um die verschiedenen Möglichkeiten des Bauablaufs, die Ressourcenplanung und die $B$ austelleneinrichtung zu prüfen und zu bewerten [9]. A ußerdem erschweren die heterogenen Strukturen und die Vielzahl kleiner Unternehmen in der Bauwirtschaft die systematische Logistikplanung auf B austellen und gleichzeitig die detaillierte V orbereitung und sinnvolle U msetzung der B austellenlogistik [10].

Zwar ist mittlerweile die digitale BIM -basierte Planung von $G$ ebäuden und $G$ roßanlagen weit verbreitet, jedoch finden Aspekte der Baustellenlogistik kaum Berücksichtigung, obwohl bereits einige wichtige Informationen zur Planung und Steuerung der B austellenlogistik in aktuellen digitalen M odellen vorhanden sind [11].

\subsection{Stand der Forschung}

In der B auindustrie setzt sich zurzeit das K onzept der Digitalen Baustelle zur Planung immer stärker durch. Zu den Bestandteilen der Digitalen Baustelle gehören die dreidimensionale Bauwerksmodellierung, die Einbindung logistischer Prozesse, eine zentrale Datenverwaltung sowie die A nimation und Simulation von V orgängen auf der B austelle [12]. So werden für die K onstruktions- und Montageplanung von Großanlagen digitale $M$ ethoden eingesetzt, um eine BIM -basierte A rbeitsweise zu realisieren. Informationen zu B auwerken, B aumaterialien, Terminen und Kosten können über K ommunikationsplattformen im gesamten $B$ auprozess allen $B$ eteiligten zur V erfügung gestellt werden [2].

Hierzu werden digitale 3-D M odelle nach dem BIM Prinzip erstellt, die von Fachabteilungen im Rahmen von Planungsaufgaben eingesetzt werden können. Neben geometrischen Informationen der A nlage enthält ein BIM Modell daher weitere Informationen wie Termine, Kosten oder auch Eigenschaften zu einzelnen A nlagenelementen [13]. Charakterisiert wird ein BIM -M odell durch die A nzahl der Informationstypen, die als Dimensionen des M odells bezeichnet werden. [14]. Die Erweiterung von dreidimensionalen CAD-M odellen mit der Projektterminplanung stellt einen Teil der sogenannten n-D-M odellierung dar. Die Grundidee ist die Erw eiterbarkeit eines 3-D-M odells um weitere Dimensionen. Mittels 4-D BIM werden die M odelle um eine Zeitdimension in Form eines Terminplans erweitert, wodurch eine K oordination der Logistikprozesse mit der dynamisch sich verändernden B austellenumgebung ermöglicht wird [15].

BIM-M odelle für die Baulogistik werden heute im H och-, Tief- und Tunnel bau eingesetzt. BIM -M odelle in der B auw irtschaft werden in der L iteratur für die B austelIeneinrichtungsplanung [16], M aterialflusslogistik [17], $B$ auprojektplanung $[6,18]$ und $B$ austellensimulation [19] angewandt. Da die ereignisdiskrete Simulation zur methodischen Absicherung der Planung, Steuerung und Ü berwachung der $M$ aterial-, Personen-, Energie- und Informationsflüsse eingesetzt wird [21], kann sie auch die Planung der B austellenlogistik sinnvoll unterstützen.

Für die Planung der Baustellenlogistik im Großanlagenbau ist ein $V$ orgehen notwendig, das die spezifischen Eigenschaften der B aubranche berücksichtigt. V or dem Hintergrund der zuvor beschriebenen Problematik wird in diesem B eitrag ein Vorgehen zur BIM -basierten Logistikplanung im Großanlagenbau und zu ihrer simulativen $A$ bsicherung vorgestellt. 


\section{BIM-basiertes Vorgehen zur Baustellenplanung}

$B$ ei der Planung der B austellenlogistik müssen nicht nur der A blauf des B auprojekts, die B auprozesse und die logistischen A ktivitäten, sondern auch die verfügbaren Ressourcen, die spezifischen Transport- und Lagerungsbedingungen für die Bauelemente sowie der $M$ aterialfluss und die Lagerkapazitäten auf der B austelle berücksichtigt werden. Eine Herausforderung besteht darin, die BIM -basierten M odelle, um Logistikbeschreibungen zu erweitern und unter den jeweiligen U mgebungsbedingungen in konkrete Logistikmodelle zu überführen.

Die $V$ orgehensweise umfasst neben der Formalisierung von produktindividuellen Logistikanforderungen auf $\mathrm{B}$ asis projektspezifischer Randbedingungen und $\mathrm{Me}$ thoden zur semi-automatischen Generierung von Logistikmodellen für die A nalyse und Planung der B austellenlogistik auch $V$ isual isierungs- und $A$ daptionskonzepte zur V erwendung der digitalen M odelle auf der B austelle. Das Zusammenwirken der einzelnen B austeine der V orgehensweise einer BIM -basierten Planung der Baustellenlogistik im $G$ roßanlagenbau ist in A bbildung 1 dargestellt. A usgehend von einem detaillierten BIM-M odell der A nlage (A nwendungsbeispiel) mit allen dazugehörigen Komponenten, zuvor definierten M ontageprozessen und Montagereihenfolgen werden im ersten Schritt A nforderungen bezüglich $B$ austellenlogistik und der eigentlichen Durchführung der Montage mit entsprechenden Ressourcen über eine 0 ntologie verknüpft. Dabei werden auch das individuelle B austellenlayout und die Ressourcen für das A nwendungsbeispiel berücksichtigt. U m auf $B$ asis der A nforderungen und dazugehörigen Informationen eine semi-automatische $G$ enerierung eines Logistikmodells (inkl. Bauanleitung) zu realisieren, werden $\mathrm{M} \mathrm{e-}$ thoden für die Erstellung des digitalen M odells mit logistikrelevanten Informationen vorgeschlagen.

Im nächsten Schritt werden die Gültigkeit des digitaIen Logistikmodells und der auf seiner Basis erstellten Bauanleitung, die zusammen die B asis für die Logistikplanung repräsentieren, simulativ überprüft. Im Laufe des Projektfortschritts auf der Baustelle können die Randbedingungen oder Prozesse verändert werden, daher müssen diejeweils neuen Informationen ebenfalls bewertet werden. Hierzu werden diese in das bestehende Logistikmodell integriert und wiederum simulationsgestützt geprüft, um Probleme oder einen A npassungsbedarf im Projektverlauf frühzeitig zu erkennen.

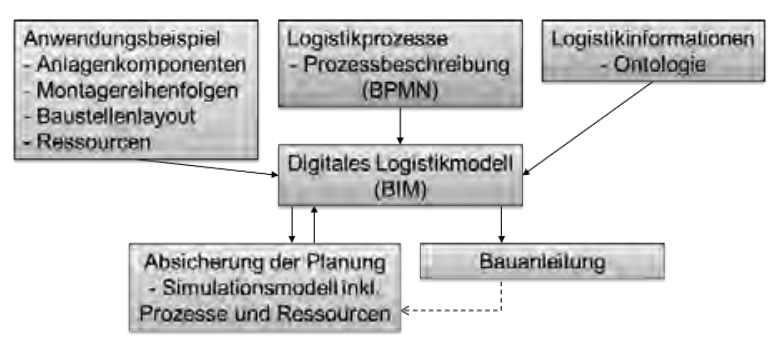

Abbildung 1: Zusammenwirken der Bausteine einer BIM-basierten Planung der Baustellenlogistik im Großanlagenbau

$N$ achfolgend werden die einzelnen B estandteile (erarbeitete 0 ntologie mit logistikrelevanten Informationen, allgemeingültig beschriebene Prozesse auf der Baustelle, Planungsszenarien, B auanleitung und Simulation) sowie ihre Rolle im Rahmen des entwickelten Vorgehens kurz vorgestellt.

\subsection{Rolle der Ontologie}

Für die Realisierung der digitalen B austellenlogistik im $G$ roßanlagenbau ist eine für die Vorgehensweise fachspezifische Ontologie entwickelt worden, um Informationen aus einem BIM -M odell, bestehend aus einer A nlage mit seinen $\mathrm{K}$ omponenten, $\mathrm{M}$ ontageprozessen und $\mathrm{M}$ ontagereihenfolgen, mit den Informationen der $B$ austellenlogistik sowie Informationen zur Durchführung der M ontage mit den dazu notwendigen logistischen Ressourcen zu verknüpfen. $D$ ie O ntologie [22] beschreibt einerseits formal die semantischen Zusammenhänge zwischen den A nforderungen an die B austellenlogistik sowie zw ischen den $\mathrm{A}$ nforderungen an $\mathrm{M}$ ontageprozesse, an den Projektgegenstand (z. B. B auelemente einer Großanlage) und an weitere relevante $O$ bjekte auf der B austelle (z. B . Lager, A nlieferungsfläche, Ressourcen usw.); sie enthält auch M ontage- und Logistikinformationen für die Gestaltung der $\mathrm{A}$ bläufe auf der Baustelle in den Planungsszenarien. A ndererseits wird die Ontologie als ein Informationsmodell verwendet, aus dem basierend auf einem detaillierten BIM-M odell einer Großanlage logistische A nforderungen abgel eitet und sogenannte $B$ auanl eitungen generiert werden können.

\subsection{Beschreibung der Logistikprozesse}

Für die Unterstützung der Logistikplanung auf der Baustelle und ausgehend von den A nforderungen im G roßanlagenbau werden Prozessabläufe für die Baustellenlogistikplanung mittels der M odellierungssprache B PM N 
(B usiness Process M odeling L anguage) als R eferenzprozesse dargestellt, mit denen die B eispielabläufe auf $\mathrm{B}$ austellen (in den Planungsszenarios) abgebildet werden können. D ie definierten BPM N-R eferenzprozesse betreffen die $B$ austellenbereiche: A nlieferung, B austellenlager, $Z$ wischenlager, M ontage und Entsorgungslager. Die einzelnen Referenzprozesse beinhalten logistikbezogene Tätigkeiten auf der B austelle, wie das Transportieren, Lagern, Puffern, Entpacken, U mschlagen, Prüfen und K ommissionieren von Baumaterialien und -elementen. A uf $B$ asis der dokumentierten B PM N-R eferenzprozesse wird die Erstellung des digitalen Logistikmodells sowie des Simulationsmodells umgesetzt und somit eine detailliertere Planung von Prozessen auf der B austelle ermöglicht.

\subsection{Rolle des BIM-basierten Logistikmodells}

Das BIM -basierte Logistikmodell wird unter Verwendung eines konkreten BIM-A nwendungsbeispiels (inkl. A nlagekomponenten, M ontagereihenfolgen, B austellenlayout und geplanten Ressourcen) semi-automatisch erstellt. Das Logistikmodell beinhaltet neben den projektspezifischen Informationen und Geometriedaten der A nlage auch logistikrelevante Informationen aus der fachspezifischen Ontologie. In diesem Logistikmodell können nicht nur Termine für die einzelnen Bau- und Logistikprozesse gesichert werden, sondern es kann auch für die Erstellung einer B auanleitung verwendet werden. Das L ogistikmodell (inkl. B auanleitung) stellt die Grundlage für die Erstellung von Planungsszenarien dar, die im Rahmen der BIM -basierten Planung der B austel lenlogistik im $\mathrm{G}$ roßanlagenbau simulativ abgesichert werden.

\subsection{Rolle der Bauanleitung}

In 4-D BIM -M odellen ist zumeist nur eine grobe zeitliche $A$ bfolge der einzelnen Bauabschnitte abgebildet, bei der beispiel sw eise alle B auelemente eines $G$ ew erks in einer Etage innerhalb einer festgelegten Woche eingebaut werden. Für eine genaue Planung der Logistikprozesse wird jedoch eine präzisiere Zeitplanung benötigt. Da der A ufwand für die manuelle Erzeugung einer Schritt-fürSchritt-B auplanung auf individueller B auteilebene nicht wirtschaftlich ist, wird dieser Prozess automatisiert. Hierzu werden Regeln zur M ontage der B auelemente definiert. Diese Regeln beschreiben die A bhängigkeit der B auel emente voneinander und ermöglichen - in K ombination mit Informationen über die $L$ age der Elemente relativ zueinander - die semi-automatische Generierung einer Schritt-für-Schritt-B auanleitung. M it dieser lässt sich schließlich die genaue A bfolge der korrespondierenden Logistikprozesse festlegen. Durch die Betrachtung und Einreihung jedes einzelnen $B$ auelements wird so bei $B$ edarf eine Terminplanung für jedes einzelne $B$ auelement ermöglicht.

\subsection{Erstellung der Planungsszenarien}

A uf $B$ asis der definierten BPM N-Referenzprozesse, eines BIM -basierten Logistikmodells, einer Bauanleitung und dazugehörigen Informationen aus der Ontologie kann eine Planung der Prozesse auf der B austelle durchgeführt werden. Hierzu werden Planungsszenarien definiert. Ein Planungsszenario enthält die terminliche Anordnung der M ontage- und Logistikprozesse sowie Anlieferungen von $B$ aumaterialien unter B erücksichtigung der für ein Anwendungsbeispiel geplanten Ressourcen und dessen $L$ ayouts. Dabei werden verschiedene Szenarien betrachtet und somit auch Zustände einer B austelle definiert, sodass relevante Eigenschaften sowohl der B au- und Logistikaktivitäten als auch der Bauelemente und -materialien des Bauprojekts berücksichtigt werden können. B eim Erstellen der Szenarien werden insbesondere die von der Bauanleitung vorgeschlagenen Reihenfolgen der M ontage und die Zeitpunkte der A nlieferungen von Baumaterialien berücksichtigt und aufeinander abgestimmt. Zur A usgestaltung der Prozesse auf der Baustelle werden die Informationen aus der Ontologie (z. B. A nforderungen der $B$ aumaterialien für die $L$ agerung und den Transport auf der Baustelle) und die BPM N-Referenzprozesse verwendet. Die für ein Projekt geplanten Ressourcen, das Personal und das entsprechende L ayout sowie die definierten Planungsszenarien werden dann mit Hilfe eines mit dem Simulationswerkzeug A nyL ogic entwickelten Simulationsmodells hinsichtlich ihrer Gültigkeit überprüft.

\subsection{Rolle der Simulation}

Zur Sicherstellung der Gültigkeit des Logistikmodells werden die im BIM -M odell aufgeführten Reihenfolgen, Restriktionen und verwendeten Ressourcen überprüft. $\mathrm{Hierzu}$ wird ein $\mathrm{M}$ aterialflusssimulationsmodell auf $\mathrm{Ba}$ sis der Planungsdaten erstellt, das die in den Planungsszenarien definierten Prozesse (wie Reihenfolge der M ontage, Transporte und Lagerungen) sowie Nutzung der verfügbaren Ressourcen (wie Lagerflächen und Transportmittel) modelliert und die ausgewählten Planungsszenarien experimentell untersucht. $\mathrm{Nach}$ der simulationsgestützten Überprüfung liegt ein konsistentes 
und valides L ogistikmodell vor, das auch zur Steuerung der Baustellenlogistik verwendet werden kann. Darüber hinaus liefert das Simulationsmodell bereits vor B eginn der eigentlichen Bautätigkeiten Leistungsdaten für eine mit entsprechender $M$ aterialfluss- und L agertechnik vorgegebenen A usstattung der Baustelle. Mit Hilfe der Simulation können somit die A nforderungen an die Dimensionierung der Lagerflächen auf der Baustelle überprüft und der E insatz des Personals und der Ressourcen für die Real isierung des Bauprojekts quantifiziert werden. A ußerdem wird es möglich, die auf der B austelle geplanten logistischen Ressourcen über einen definierten Zeitraum zu überprüfen (u. a. A uslastung von Transportmitteln, z. B. von K ränen und Gabelstaplern, B elegung von Lagerflächen sowie Personaleinsatz).

Im Rahmen der BIM -basierten Planung der Baustellenlogistik wird das jeweils anwendungsspezifische Simulationsmodell zur A bsicherung der Planung basierend auf den Daten der Referenzprozesse, Ontologie und BIM-Logistikmodelle standardisiert aufgebaut. Dieses V orgehen ist auch als B asis für zukünftige Bauprojekte wiederzuverwenden.

\section{Anwendungsbeispiel}

In diesem Abschnitt wird die U msetzung des BIM -basierten Vorgehens auszugsweise an einem A nwendungsbeispiel vorgestellt. In diesem Zusammenhang wird zunächst das A nwendungsbeispiel vorgestellt und anschlieBend wird auf die Simulation zur A bsicherung der durchgeführten B austellenlogistikplanung eingegangen. $A b$ schließend werden die Simulationsergebnisse diskutiert.

\subsection{BIM-Modell}

Zur nachvollziehbaren U msetzung wird ein einfaches BIM -M odell verwendet. Da M odelle aus der Praxis in der Regel sehr komplex sind, wird ein kleines StahlbauM odell (vgl. A bbildung 2) konstruiert. Dieses besteht aus zwei L agerflächen und $78 \mathrm{~B}$ auelementen, die trotz ihrer geringen K omplexität einem real en Stahl bau-M odell entsprechen. Die K onstruktion setzt sich aus einem Fundament und einer darauf befindlichen Stahlbaukonstruktion zusammen. Die Stahlkonstruktion besteht aus vier Stützen, fünf Trägern, darauf liegend zwei B etonplatten, 60 Bolzenverbindungen in 30 Gruppen, 16 Stahlwinkeln und 16 A nkerstäben. Darüber hinaus werden in der Vorfertigung Fußplatten mit den Stützen und beidseitig Stirnplattenanschlüsse an einen der Träger geschweißt.
Die im M odell verwendeten B olzen haben den gleichen D urchmesser, um die L agerhaltung zu vereinfachen [23].

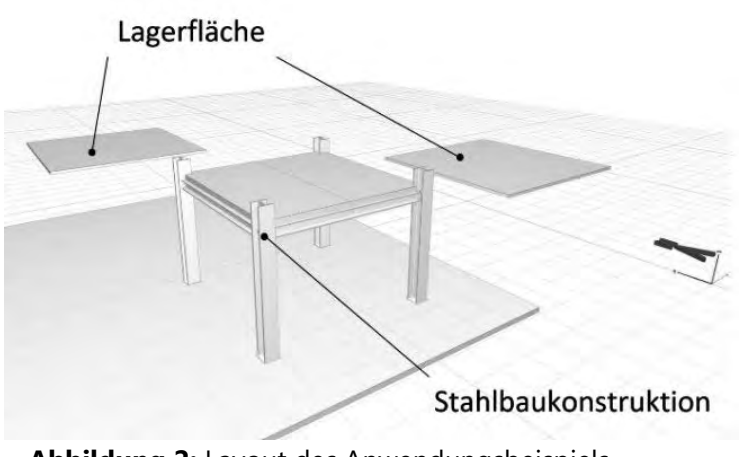

Abbildung 2: Layout des Anwendungsbeispiels

\subsection{Durchführung der Logistikplanung}

Für die Planung der zugehörigen Prozesse auf der $\mathrm{B}$ austelle wird zunächst auf B asis des BIM -L ogistikmodells zum A nwendungsbeispiel eine Bauanleitung semi-automatisch erstellt. Mittels dieser Bauanleitung werden sechs aufeinanderfolgende B auprozesse (Errichtung von vier Stützen, Einbau von Trägern und Geschossdecke (bestehend aus zwei B etonplatten)) definiert. Dabei wird der A ufbau des Fundaments nicht berücksichtigt, da bei dem Anwendungsbeispiel ausschließlich Bauprozesse der Stahlkonstruktion betrachtet werden. Um die Logistikplanung durchführen zu können, werden Annahmen bezüglich der $A$ nlieferungen der $B$ auteile getroffen. Insgesamt werden drei Anlieferungen geplant (zwei A nlieferungen für die Stahlträger und Stahlstützen sowie eine A nlieferung der Geschossdecke), die A nlieferungen sollen in Abhängigkeit von definierten Szenarien vor dem B eginn der M ontageprozesse erfolgen. Für die Definition der Planungsszenarien werden auch A nnahmen bezüglich der auf der B austelle zur Verfügung stehenden Ressourcen und Baustellenmitarbeiter getroffen. Somit werden für das Anwendungsbeispiel zwei Planungsszenarien ausgewählt. Im ersten beispiel haften Planungsszenario sind ein $\mathrm{K}$ ran und zwei B austellenmitarbeiter (ein $M$ onteur und ein $\mathrm{K}$ ranführer) eingeplant. Die sechs $M$ ontageprozesse werden hier gemäß der Bauanleitung nacheinander ausgeführt, die drei A nlieferungen der Bauteile erfolgen durch Lieferanten; alle B auteile werden vor ihrer $M$ ontage zwischengelagert und mit dem $\mathrm{K}$ ran zum M ontageort transportiert und montiert. Die A nforderungen an die Transporte und Lagerungen der einzelnen $B$ auelemente werden gemäß der Ontologie definiert. Im zweiten Planungsszenario sollen die Montageprozesse der Stahlstützen parallelisiert werden ( $d$. h., es werden jeweils zwei Stützen gleichzeitig montiert); anschließend 
werden, wie im ersten Szenario, Stahlträger und Geschossdecke nacheinander aufgebaut. Die A nlieferungen der B auelemente erfolgen auch hier vor der $M$ ontage der $B$ auelemente zur $A$ nlief erungsfläche, anschließend werden die Bauelemente je nach Bedarf gelagert. Um das zweite Planungsszenario zu realisieren, wird zusätzlich zu den eingeplanten Ressourcen aus dem ersten Planungsszenario ein zweiter $\mathrm{K}$ ran eingeplant.

A us dem BIM -M odell und dem dazugehörigen Layout werden die einzelnen Positionen der Objekte, Lagerflächen und M ontageorte auf der B austelle und die $L$ ängen der Transportwege zwischen diesen Positionen bestimmt. Für das A nwendungsbeispiel wird neben einer A nlieferungsfläche auch ein offenes $B$ austellenlager eingeplant. A uf dieser Basis können die Zeiten der Transporte auf der B austelle berechnet werden. Unter B erücksichtigung der projektrelevanten Termine, der M ontagereihenfolge und der logistischen Abläufe werden die zwei ausgewählten Szenarien in Form von Gantt-Diagrammen dargestellt. In den Planungsszenarien werden die einzelnen Termine der geplanten A bläufe auf der B austelle (Transporte, Lagerungen und M ontagen) und die L iefertermine koordiniert. Somit kann mittels der Planungsszenarien die Projektdauer bis zur Fertigstellung der Großanlage näherungsweise bestimmt werden. Für die Gestaltung der Transporte und Lagerungen werden u. a. Informationen zu M aterial- und R essourcenanforderungen für jeden Prozess (z. B. Lagerbedingungen für Stahlträger) aus der fachspezifischen Ontologie verwendet.

\subsection{Durchführung der Simulation}

In der Simulation werden die in den Szenarien geplanten Prozesse (Logistik- und M ontageprozesse) und der Einsatz verschiedener Ressourcen auf der Baustelle überprüft. Das Simulationsmodell berücksichtigt hierfür Personal, A usführungszeiten, $M$ aterial und Ressourcen. Für seine Erstellung werden als Eingangsdaten der Terminund A blaufplan (Startpunkt, Dauer und Reihenfolge der Prozesse), die Anlieferungstermine, die Material- und Ressourcenanforderungen für jeden Prozess sowie die A ngaben für die Gestaltung der B auprozesse, Warenannahme und Transportvorgänge verwendet. Ein gemäß dem Terminplan generierter $A$ uftrag durchläuft während der Simulation einen in einem Planungsszenario definierten Prozess ( $z$. B. W arenannahme, Lagerungen, Transporte und M ontage). Die A ufträge enthalten die notwen- digen Informationen, um jeden Prozess richtig und vollständig abzubilden (z. B. Ressourcenanforderungen, Lademenge, Stückliste der B auprozesse, Startzeitpunkt und Dauer).

Die Prozessabläufe (K rantransporte, A nlieferung auf der $B$ austelle sowie $M$ ontage und $V$ ormontage) werden als R eferenzprozesse im Simulationsmodell nachgebildet und laufen während der Simulation entsprechend der V orgaben in dem definierten Planungsszenario ab. Die modellierten R eferenzprozesse entsprechen einer allgemeingültigen Prozessbeschreibung auf der Baustelle (BPM N-Referenzprozesse). Sie können somit auch bei neuen Bauprojekten und Planungsszenarien erweitert, angepasst und wiederverwendet werden.

Im Simulationsmodell (vgl. A bbildung 3) werden auch das L ayout, die Positionen der sechs M ontageorte der einzelnen Bauelemente und die relevanten Prozesse (als $W$ arteschl angen für die M ontageprozesse im B ereich „Processes" und für die Lagerungen im Bereich "Storages“) nachgebildet. Zudem werden als Ressourcen im ersten Planungsszenario ein K ran, ein $\mathrm{K}$ ranführer und ein A rbeiter hinterlegt, die bei B edarf (bspw. im zweiten Planungsszenario) um weitere Ressourcen (bspw. um einen zweiten $\mathrm{K}$ ran) ergänzt werden können. Die Planungsdaten, wie die Reihenfolge der Lieferungen, der M ontage, der L ager- und Transportprozesse, die Stücklisten für jeden Bauprozess, die bei den Prozessen verwendeten Ressourcen, die geplanten A nkunftszeiten der Lieferungen auf der Baustelle sowie die geplanten Startzeitpunkte der $B$ auprozesse, werden in Form von definierten Tabellen in dem Simulationsmodell implementiert. Nach Durchführung der Simulationsläufe können die Planungszeiten (Start- und Endzeitpunkte der Bauprozesse in der Projektplanung) und die simulierten Zeiten der M ontageprozesse verglichen werden. Mit Hilfe der Simulation wird sichergestellt, dass die Komponenten nur dann geliefert werden, wenn sie bedarfsgerecht gelagert, transportiert oder direkt eingebaut werden können. Somit wird während der Simulation geprüft, ob die für ein Bauprojekt eingeplanten Ressourcen für die Fertigstellung der Anlage ausreichen.

Im Fall eines Fehlers im Planungsszenario wird während der Simulation eine Fehlermeldung ausgegeben (z. B., dass ein M ontageprozess nicht ausgeführt werden kann, da die notwendige Ressource nicht frei ist). 


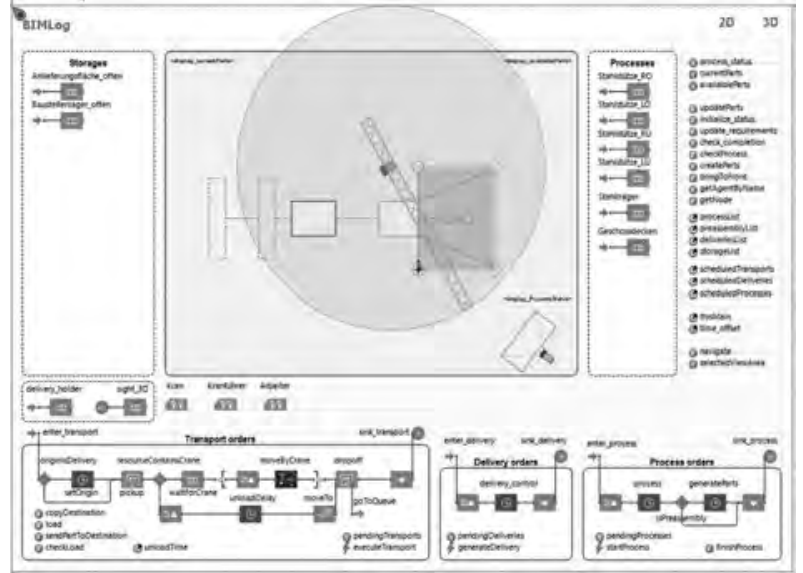

Abbildung 3: Simulationsmodell in AnyLogic

Die simulierten Zeiten der Montageprozesse werden während der Simulation in einer Tabelle eingetragen. Somit können die Dauer der einzelnen B auprozesse (in A bstimmung mit den simulierten Transport- und L agerprozessen) und die gesamte Projektdauer näherungsweise bestimmt werden. A uf diese Weise wird simulativ die $B$ auanleitung überprüft. Die A nimation der Prozesse verdeutlicht den simulierten A blauf der A nlieferungen der $B$ auelemente, der M ontage- und Logistikprozesse (inkl. Transportvorgänge und Lagerungen der B auelemente) auf der Baustelle. Zur B ewertung der Planungsszenarien (z. B. 1. Planungsszenario mit einem $\mathrm{K}$ ran und 2. Planungsszenario mit zwei $K$ ränen) werden neben den simulierten Prozesszeiten auch L eistungskennzahlen wie A uslastung der Ressourcen (in Prozent) und A uslastung der L agerflächen (in L agereinheiten) über die gesamte D auer des Projekts protokolliert (siehe A bbildung 4 für das erste Planungsszenario).

\subsection{Simulationsergebnisse}

D urch die Simulation der beiden oben beschriebenen Planungsszenarien werden nicht nur die geplanten Termine und A nnahmen überprüft, sondern auch die A bstimmung der $M$ ontagereihenfolge mit den logistischen Prozessen validiert. Dabei werden die tatsächlichen Start- und Endzeitpunkte der M ontageprozesse in T abellen festgehal ten (für das erste Planungsszenario mit einem $\mathrm{K}$ ran beträgt die simulierte Projektdauer 118,3 Stunden, für das zweite Planungsszenario mit zwei $\mathrm{K}$ ränen beträgt die simulierte Projektdauer insgesamt 93,3 Stunden) und die L ager- und Ressourcenauslastungen ausgewertet. A uf dieser Basis können die geplanten Planungsszenarien angepasst und ggf. verbessert werden.

Die Diagramme zur A uslastung der Ressourcen und
A rbeiter (A bbildung 4) verdeutlichen, dass beim ersten simulierten Planungsszenario der $\mathrm{K}$ ran und der K ranführer vor allem zu Projektbeginn fast immer vollständig ausgelastet sind. B ei der A uslastung der L agerflächen ist erkennbar, dass am A nfang der B auprozesse die A nlieferungsfläche eine hohe A uslastung hat und dass der Bestand im L ager über die betrachtete Projektdauer sehr gering ist (maximal drei L adeeinheiten).
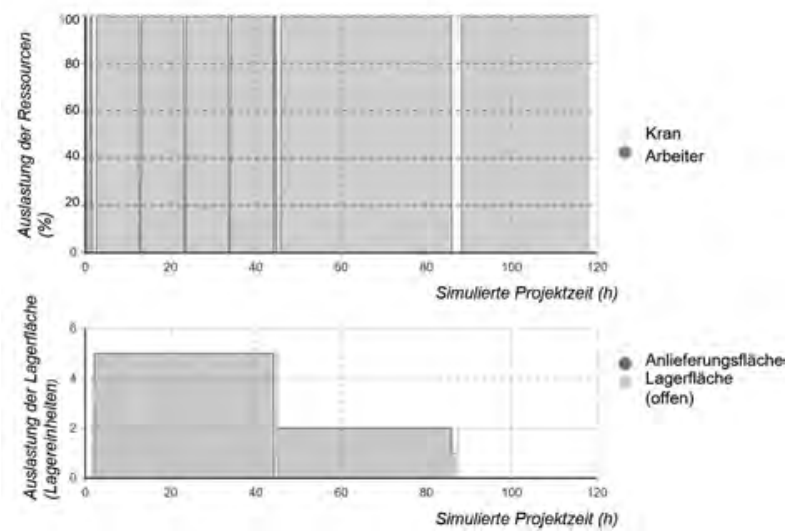

Abbildung 4: Ausgewählte Simulationsergebnisse

Bei dem zweiten Planungsszenario wird durch den Einsatz von zwei K ränen auf der Baustelle die A uslastung der einzelnen $\mathrm{K}$ räne über die Projektdauer reduziert. A Ilerdings ist die $\mathrm{K}$ ollisionsprüfung der beiden $\mathrm{K}$ räne bei einer Logistiksimulation nicht Gegenstand der B etrachtung; dazu müssen entsprechende 3-D K ollisionsuntersuchungen durchgeführt werden. Die A uslastung der Lagerfläche ist ähnlich niedrig wie im ersten Szenario, sodass die Dimensionierung der Lagerflächen bei beiden Szenarien gleichbleibt; allerdings kann die Projektdauer im zweiten Planungsszenario gegenüber dem ersten Szenario reduziert werden.

\section{Zusammenfassung und Ausblick}

Der Beitrag stellt eine BIM-basierte Vorgehensweise vor, mit der eine digitale Planung der B austellenlogistik realisiert werden kann. Bei der Planung wird insbesondere der Zusammenhang zwischen der M ontage und L 0 gistik der einzelnen $B$ auelemente und -materialien berücksichtigt. Dazu wird das logistikrelevante Planungswissen in einer $O$ ntologie modelliert. M it den Informationen aus dieser Ontologie können BIM -M odelle erweitert werden. Die für diese BIM -M odelle erstellten Bauanleitungen bilden die Basis für die Erstellung von Planungsszenarien. M it der simulationsgestützten Ü berprüfung dieser Planungsszenarien liegt ein konsistentes und 
valides B IM -basiertes L ogistikmodell vor; dieses M odell kann auch als Basis für die Steuerung der Logistik auf der $B$ austelle verw endet werden.

In den obigen Untersuchungen zum A nwendungsbeispiel wird die Simulation nur zur A bsicherung von Planungsszenarien mit festgelegten Transportaufträgen verwendet. Die Evaluation des BIM -basierten V orgehens zur B austellenplanung hat jedoch gezeigt, dass es sinnvoll sein kann, das Simulationsmodell so zu erweitern, dass es auch für eine umfangreichere Planung der Logistikprozesse auf der B austelle einsetzbar wird.

Zukünftig werden auf Grundlage der erzielten Ergebnisse weitere Forschungen zum Thema digitale Planung und Steuerung der Baustellenlogistik durchgeführt. Ein wichtiges Forschungsziel ist in diesem Zusammenhang die automatische Erstellung von Logistikmodellen für die Baustelle und ihre semi-automatische $V$ alidierung mittels Simulation.

Danksagung. Dieser Beitrag entstand im Rahmen des Forschungsprojekts „BIM Log - BIM -basierte Logistikplanung und -steuerung im Großanlagenbau", das unter der IG F-Vorhaben-N ummer $19720 \mathrm{~N}$ der B undesvereinigung Logistik (BVL) geführt und über die Allianz industrieller Forschung (A iF) im R ahmen des Programms zur Förderung der Industriellen Gemeinschaftsforschung (IGF) vom Bundesministerium für Wirtschaft und Energie (BM Wi) aufgrund eines $B$ eschlusses des Deutschen $B$ undestages gefördert wird.

\section{Literatur}

[1] Schuh G, Hering N, B runner A. Einführung in das L Ogistikmanagement. In Schuh G., Stich V., editors. L 0gistikmanagement. $H$ andbuch $P$ roduktion und $M$ anagement. 2 A uflage. B erlin: Springer; 2013. S. 1-33.

[2] Schach R, Schubert N. L ogistik im Bauwesen. Wissenschaftliche Zeitschrift der Technischen U niver sität D resden. 2009; 58(1-2): 59-63.

[3] Wenzel W, Gliem D, L aroque C, Kusturica W. Sichere Prognose der Dauer logistischer Prozesse. Industrie 4.0 M anagement. 2018; 34(5): 43-46.

[4] K ügler M . CA D-integrierte M odellierung von agentenbasierten Simulationsmodellen für die B auablaufsimulation im Hochbau. Kassel: Kassel U niversity Press. 2012.

[5] Bernd F. Die Entwicklung projekt- und fertigungsspezifischer B aul ogistikprozesse. In Volkhard F., editor. Simulation von U nikatprozessen - Neue Anwendungen aus F orschung und Praxis. Kassel: $K$ assel University Press; 2011. S. 45-62.

[6] Liu H, A I-Hussein M , L u M . BIM -based integrated approach for detailed construction scheduling under resource constraints. Automation in Construction. 2015; 53: $29-43$.
[7] Horenburg T. Simulationsgestützte A blaufplanung unter Berücksichtigung aktueller Baufortschrittsinformationen. M ünchen: Technische U niversität M ünchen. 2014.

[8] Kalusche W. Projektmanagement für B auherren und Planer. M ünchen: Oldenbourg Wissenschaftsverlag. 2012.

[9] Hofstadler C. Bauablaufplanung und Logistik im B aubetrieb. Berlin: Springer. 2007.

[10] Weber J. Simulation von L ogistikprozessen auf B austellen auf $B$ asis von 3D-CAD Daten. Dortmund: Universitätsbibliothek Technische U niversität D ortmund. 2007.

[11] W hitlock K, A banda FH, M anjia M B, Pettang C, Nkeng GE. BIM for Construction Site L ogistics M anagement. J ournal of Engineering, Project \& Production Mangement. 2018; 8(1): 47-55.

[12] Baumgärtel T, Borrmann A, Günthner WA, Juli R, K laubert $C$, L ederhofer $E, M$ ack J, Will berg U. B auen heute und morgen. In Günthner W., B orrmann A ., editors. Digitale Baustelle- innovativer Planen, effizienter Ausführen. Werkzeuge und M ethoden für das Bauen im 21. J ahrhundert. B erlin: Springer; 2011. S. 1-21.

[13] Borrmann, A., K önig, M., K och, C.\& B eetz, J. Building Information M odeling. Wiesbaden: Springer. 2015.

[14] Nävy J. Facility M anagement. Grundlagen, Informationstechnol ogie, Systemimplementierung, A nwendungsbeispiele. 5 A uflage. B erlin: Springer. 2018.

[15] Smith P. BIM \& the 5D Project Cost M anager. Procedia - Social and Behavioral Sciences. 2014; 119: 475-484 [https://doi.org/10.1016/j.sbspro.2014.03.053]

[16] A stour H. Entwicklung eines BIM -basierten Systems zur Entscheidungsunterstützung mittels Simulation für die Baustelleneinrichtungsplanung. Kassel: Kassel University Press. 2015.

[17] Cheng J CP, Kumar S. A BIM -B ased Framework for M aterial L ogistics Planning. In Seppänen O., G onzález V.A., A rroyo P., editors. 23rd Annual Conference of the International Group for Lean Construction; 2015. Perth, A ustralia. S. 33-42.

[18] Kim H, A nderson K, Lee S, Hildreth J. Generating construction schedules through automatic data extraction using open BIM (building information modeling) technology. Automation in Construction. 2013; 35: 285-295.

[19] Song S, Yang J, Kim N. Development of a BIM -based structural framew ork optimization and simulation system for building construction. Construction Innovation. 2012; 63(9): 895-912.

[20] Schober K.-S., H off P. Think act. B eyond M ainstream: Digitalisierung in der Bauwirtschaft.: Der europäische Weg zur Digital isierung; 2016. URL: https://www.rolandberger.com/publications/publication_pdf/roland_berger_digitalisierung_bauwirtschaft_final.pdf.

[21] Wenzel S. Simulation logistischer Systeme. In Tempelmeier $\mathrm{H}$., editor. M odellierung logistischer Systeme. Berlin, H eidelberg: Springer; 2018. S. 1-34.

[22] Wenzel S, Stolipin J, Weber J, K önig M. Digitale Planung der Baustellenlogistik im Großanlagenbau Ontologie zur N utzung digitaler M odelle für die L ogistikplanung auf der B austelle. Industrie 4.0 M anagement. 2019; (3): $55-59$.

[23] Lohse W, Laumann J, Wolf C. Stahlbau 1: Bemessung von Stahlbauten nach Eurocode mit zahl reichen Beispielen. Springer-Verlag. 2016. 